\title{
Enhanced recovery pathway versus standard care in patients undergoing video-assisted thoracoscopic lobectomy
}

\author{
Alessandro Brunelli, MD, ${ }^{\mathrm{a}}$ Caroline Thomas, MD, ${ }^{\mathrm{b}}$ Padma Dinesh, BSc, ${ }^{\mathrm{a}}$ and Andrew Lumb, $\mathrm{MD}^{\mathrm{b}}$
}

\section{ABSTRACT}

Objective: The objective of this study was to compare outcomes after videoassisted thoracoscopic lobectomy or segmentectomy before and after introduction of an enhanced recovery program.

Methods: Data from 600 patients undergoing video-assisted lobectomy or segmentectomy between April 2014 and January 2017 were analyzed. A comparative analysis was performed between patients undergoing operation before (365 patients) and after ( 235 patients) the start of the enhanced recovery program. The incidence of cardiopulmonary complications and 30-day and 90-day mortality, postoperative length of stay, and 30-day and 90-day hospital readmission rates were evaluated. Risk-adjusted cardiopulmonary morbidity and 30-day mortality were calculated for each group and compared.

Results: The 2 groups had a similar postoperative length of stay (enhanced recovery pathway median 5 days vs pre-enhanced recovery pathway $4, P=.44$ ), cardiopulmonary complication rates (enhanced recovery pathway $22.6 \%$ vs preenhanced recovery pathway $22.4 \%, P=.98$ ), 30-day mortality rates (enhanced recovery pathway $3.8 \%$ vs pre-enhanced recovery pathway $2.2 \%, P=.31$ ), and 90-day mortality rates (enhanced recovery pathway $4.7 \%$ vs pre-enhanced recovery pathway $3.0 \%, P=.37$ ). No differences were noted in terms of 30 day (enhanced recovery pathway $7.2 \%$ vs pre-enhanced recovery pathway $7.4 \%, P=.94$ ) or 90 -day readmission rates (enhanced recovery pathway $9.8 \%$ vs pre-enhanced recovery pathway $12.3 \%, P=.34$ ). The risk-adjusted cardiopulmonary morbidity rates were similar in the 2 periods $(P=.76)$, whereas the riskadjusted 30-day mortality was higher in the enhanced recovery pathway period compared with the pre-enhanced recovery pathway mortality $(P=.0004)$.

Conclusions: We found no benefit conferred by the enhanced recovery program on outcomes such as cardiopulmonary complications, 30- and 90-day mortality, length of stay, and readmissions. Enhanced recovery program elements may be insufficiently different than previous standards of perioperative care to confer detectable benefits in our settings. (J Thorac Cardiovasc Surg 2017;154:2084-90)

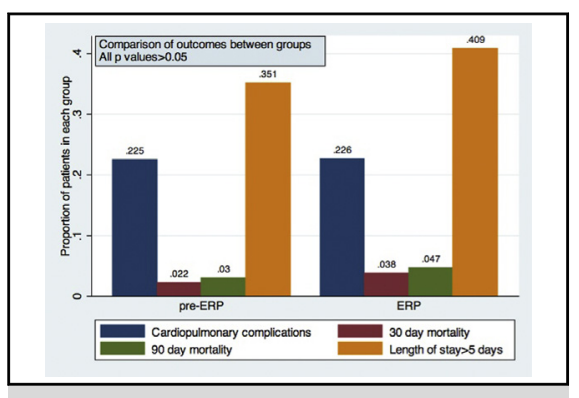

Bar graph displaying the incidence of cardiopulmonary complications, 30-day mortality, 90-day mortality, and prolonged length of stay ( $>5$ days) in each group (ERP vs pre-ERP). The differences of outcomes between groups were not significant $(P>.05)$

\section{Central Message}

The ERP does not confer an outcome benefit in patients undergoing video-assisted thoracoscopic lobectomy when part of the elements are already embedded in standard care.

\section{Perspective}

Evidence of the effect of ERP in thoracic surgery is scant. We found no outcome benefit after its introduction in our unit. Our findings raise the question whether the cost to implement the ERP for video-assisted thoracoscopic lung resections is justified. Standard care contains most of the elements traditionally included in this program.

See Editorial Commentary page 2091

See Editorial page 2082.
The enhanced recovery pathway (ERP) is a multimodal, evidence-based bundles of interventions aimed at hastening

From the Departments of ${ }^{\mathrm{a}}$ Thoracic Surgery and ${ }^{\mathrm{b}}$ Anaesthesia, St James's University Hospital, Leeds Teaching Hospital National Health Service Trust, Leeds, United Kingdom.

Received for publication March 31, 2017; revisions received June 9, 2017; accepted for publication June 16, 2017; available ahead of print July 18, 2017.

Address for reprints: Alessandro Brunelli, MD, Department of Thoracic Surgery, St James's University Hospital, Bexley Wing, Beckett St, Leeds LS9 7TF, United Kingdom (E-mail: alexit_2000@yahoo.com).

0022-5223/\$36.00

Copyright (c) 2017 by The American Association for Thoracic Surgery

http://dx.doi.org/10.1016/j.jtcvs.2017.06.037 postoperative recovery, reducing complications, shortening hospital stay, and improving patient experience.

The ERP is based on the concept of "marginal gains," based on the standardized application of multiple elements covering all aspects of perioperative care. These elements

Scanning this QR code will take you to the article title page. 


\section{Abbreviations and Acronyms \\ $\mathrm{ERP}=$ enhanced recovery pathway \\ VATS $=$ video-assisted thoracoscopic surgery}

may have a limited effect on the outcome when used in isolation, but they act synergistically to reduce surgical stress and facilitate recovery. ${ }^{1}$

The ERP has been popularized in colorectal surgery, where it has shown the greatest benefit compared with standard care, and has been shown to improve outcomes in almost all major surgical specialties., ${ }^{2,3}$ However, in thoracic surgery, there is limited and generally lowquality evidence regarding the influence of the ERP on outcome. A recent systematic review identified 6 studies, of which only 1 was a randomized trial. This review found that the ERP in elective lung surgery was associated with shorter hospital stay but similar complication, mortality, and readmission rates compared with standard care. ${ }^{4}$ In contrast to the ERP in colorectal surgery, many elements of the ERP are routinely used in thoracic surgery and are well embedded in practice as standard care (eg, fluid restriction, early mobilization, rehabilitation, early as possible enteral feeding, and the approach to pain management). Therefore, the ERP in thoracic surgery may not be dissimilar enough from standard care to improve the outcome. ${ }^{5}$

Furthermore, most of the studies dealing with the ERP in thoracic surgery did not include patients undergoing videoassisted thoracoscopic surgery (VATS) or were conducted before the widespread use of this approach. ${ }^{6-9}$ Minimally invasive surgery is a key element of the ERP in other specialties. ${ }^{10}$ VATS has been shown to reduce complications and shorten hospital stay after lung resection ${ }^{11-13}$ and conceptually seems to be the ideal surgical approach in the context of the ERP to improve the outcome. However, there is no study specifically analyzing whether the application of the ERP in the context of minimally invasive thoracic surgery would provide outcome benefits compared with standard care. A recent systematic review ${ }^{4}$ acknowledged this knowledge gap and concluded that future research is needed to investigate this aspect.

A formal ERP recently has been implemented for patients undergoing lung resection for lung cancer in our unit. The majority underwent VATS resection. Therefore, the purpose of this analysis was to assess the impact of the ERP on the outcome of patients undergoing VATS lobectomy compared with similar patients undergoing operation before the start of the ERP in our unit.

\section{PATIENTS AND METHODS}

This is a retrospective analysis performed on a prospectively maintained quality-improvement institutional database. The analysis included 600 patients undergoing VATS lobectomy (561) or VATS anatomic segmentectomies (39) between April 2014 and January 2017. These patients represent $85 \%$ of all lobectomies and segmentectomies performed at St James's University Hospital during this time period (15\% were performed through an open approach and were not included in this analysis). Sixty-seven patients whose surgery commenced via a VATS approach but who subsequently underwent conversion to open surgery for oncologic, anatomic, or technical reasons or because of intraoperative complications were included in the analysis.

The study was reviewed by the Research and Innovation Department of our hospital, which waived National Health Service Research Ethics Committee review and classified it as a service evaluation. Patients were selected for operation according to current functional guidelines. ${ }^{14}$

All operations were undertaken by board-certified thoracic surgeons using a 2- to 3-port VATS anterior approach. All patients were extubated in the operating room and transferred to a level 2 care unit (thoracic intermediate care unit) for monitoring. On the morning of the first postoperative day (day 1), patients were stepped down to a dedicated thoracic surgery ward unless clinically contraindicated.

An ERP was implemented in our unit in January 2016. For the purpose of auditing the results of the ERP, we allowed a 1-month washout period to ensure that all elements of the ERP were fully implemented and adherence to the protocol was optimized. From February 2016 to January 2017, 235 patients underwent VATS lobectomy and were managed according to the ERP program.

Elements of care that were already part of standard care before the introduction of the ERP include the following:

- Multimodal analgesia. A variable regimen for postoperative analgesia achieved with a combination of systemic (intravenous) opioid (via a patient-controlled analgesia system) and paravertebral analgesia using a $0.5 \%$ bupivacaine bolus followed by $0.25 \%$ bupivacaine postoperative infusion. Delivery of analgesia is titrated to achieve numeric pain score lower than 3 on a scale of 0 (no pain) to 10 (excruciating pain).

- Mobilization. All patients are mobilized as early as possible, most frequently on postoperative day 1 (the morning after surgery). They receive postoperative chest physiotherapy and physical rehabilitation.

- Chest drain management. All patients undergoing VATS are managed by 1 chest drain connected to a digital chest drainage system. Chest drains are removed as soon as possible when the drain output in a 24-hour period is less than $400 \mathrm{~mL}$ and air flow is less than $20 \mathrm{~mL}$ per minute for more than 6 hours without an air leak spikes.

- Other interventions include antibiotic prophylaxis (at anesthetic induction and 2 doses postoperatively); minimally invasive (VATS) surgery, use of short-acting anesthetic agents; use of nerve or paravertebral blocks as part of multimodal analgesia; avoidance of fluid overload and postoperative information provided at discharge and a telephone follow-up performed at 3 and 7 days postoperatively.

With the introduction of the ERP in January 2016, new elements of care were added. The newly introduced elements are shown in detail in Table 1. Major new components included the following:

- The development of a new patient information program. This is a perioperative ERP-focused education session for patients, which they are encouraged to attend. An explanation of the elements of the ERP is given, and the concept of the active role of the patient is introduced. At this session, patients receive a written information package and a patient diary. Before the ERP, an education session did take place but without an ERP focus and without a theme of active patient involvement.

- Incentive spirometry, including written instructions on its use to commence before surgery and to continue into the postoperative period.

- Preoperative carbohydrate loading.

- Preoperative warming commencing 45 minutes before surgery.

- Motivational talks given by the ERP nurse both preoperatively and on each postoperative inpatient day. 
TABLE 1. Changes to the standard pre-enhanced recovery pathway care introduced as the enhanced recovery pathway for thoracic surgery introduced in January 2016

Element

ERP

\begin{tabular}{|c|c|}
\hline \multicolumn{2}{|l|}{ Preoperative } \\
\hline Patient information and expectations managed & $\begin{array}{l}\text { - 2-h patient information program with emphasis on self-help } \\
\text { - ERP explained to individual patients: principle of patient role, responsibility, and active } \\
\text { involvement. Elements introduced: 20-25-min session at time of booking for surgery } \\
\text { - Information pack: patient information and diary, "my calendar," "your role in your recovery" } \\
\text { incentive spirometer instruction booklet; rewrite in progress }\end{array}$ \\
\hline Preoperative health and risk assessment & $\begin{array}{l}\text { - Ethos of "preparation starts now" from booking date } \\
\text { - Incentive spirometer explained, demonstrated, and patient practice to help motivate and give a } \\
\text { sense of purpose } \\
\text { - Preoperative occupational therapist referral system developed }\end{array}$ \\
\hline Carbohydrate loading & $\begin{array}{l}6 \times 200 \mathrm{~mL} \text { bottles preoperative Nutricia drinks (Nutricia Advanced Medical Nutrition, Schiphol, } \\
\text { The Netherlands) and instruction leaflet provided: } 4 \text { to be drunk } 6 \text { PM to } 12 \text { midnight evening } \\
\text { before surgery and } 2 \text { before } 6: 30 \text { AM morning of surgery }\end{array}$ \\
\hline Preoperative warming & $\begin{array}{l}\text { - 3M Bair Paws system (Maplewood, Minn) } \\
\text { - Patient shown picture of gown and warmer, benefits explained to outpatients } \\
\text { - Warming started } 1 \mathrm{~h} \text { preoperatively, continued in prewait and anesthetic room }\end{array}$ \\
\hline No prolonged fasting & - Patients being offered clear fluids when possible \\
\hline Same-day admission & - Achieved for some patients \\
\hline \multicolumn{2}{|l|}{ Intraoperative } \\
\hline Hypothermia prevention & - Warming gown used as a blanket and converted to Bair Hugger (3M Health Care, St Paul, Minn) \\
\hline \multicolumn{2}{|l|}{ Postoperative } \\
\hline Discharge when criteria met & $\begin{array}{l}\text { - X-ray post chest drain removal, and so forth; different standards between surgeons; protocol } \\
\text { written but not completely adopted }\end{array}$ \\
\hline Early mobilization & $\begin{array}{l}\text { - Incentive spirometer } \\
\text { - Motivation talk by ERP nurse presurgery and each postoperative day they are inpatients } \\
\text { - Patient sat out and mobilized morning after operation } \\
\text { - Topaz mobile suction unit (Medela Healthcare, Baar, Switzerland) }\end{array}$ \\
\hline Early eating and drinking & $\begin{array}{l}\text { - Supplements given } 3 \times \text { per day (if not diabetic) } \\
\text { - Motivation and encouragement to eat and drink }\end{array}$ \\
\hline Nausea and vomiting prevention & - Regular antiemetics given \\
\hline Prevent fluid overload & - IVI down after ward round on first postoperative day, day 1 \\
\hline Minimal use of systemic opioids & $\begin{array}{l}\text { - PCA stopped day 1, OxyContin (Purdue Pharma LP, Stamford, Conn) PR } 10 \text { mg BID days } 1 \text { and } \\
\text { 2, dihydrocodeine day } 3 \text { onward } \\
\text { - Paravertebral removed day } 2\end{array}$ \\
\hline
\end{tabular}

$E R P$, Enhanced recovery pathway; $I V I$, intravenous infusion; $P C A$, patient-controlled analgesia; $P R$, per rectum; $B I D$, twice daily.

- Early postoperative return to oral intake including regular nutritional supplement drinks.

- Prevention of nausea and vomiting by regular administration of antiemetic drugs.

Adherence to the program was monitored by a dedicated ERP nurse assisted by the ward nurses. All patients received all the elements that were provided by the staff, for example, attendance at the patient information program, preoperative drinks, incentive spirometer, preoperative warming, offers of preoperative oral fluids, and a prescription for all the required medications. We did not record individual patient uptake of those elements about which the patients have choice, for example, if all the preoperative drinks were consumed, how often the incentive spirometer was used, the number of doses of pro re nata analgesics required, and so forth.

\section{Statistical Analysis}

Patients managed according to the ERP were compared with those managed before the introduction of the ERP. To rule out overlapping of management between periods, we excluded from the analysis those patients operated on during the first month of ERP implementation (washout period). After 1 month, all elements of the ERP were fully implemented and adherence to the program was optimized. The main end points were in-hospital or 30-day mortality, 90-day mortality, cardiovascular and pulmonary complications occurring during the hospitalization or within 30 days from operation, postoperative length of stay, and readmission rate within 30 or 90 days from operation.

For the purpose of this study, pulmonary complications include respiratory failure (reintubation at any time after operation or assisted mechanical ventilation for $>24$ hours), acute respiratory distress syndrome, pulmonary 
edema, pulmonary embolus, pneumonia (radiologic pulmonary infiltrates, fever, leukocytosis, and suggestive clinical signs and symptoms according to Centers for Disease Control and Prevention definitions), and atelectasis requiring bronchoscopy. Cardiovascular complications include the following: acute myocardial ischemia (electrocardiogram changes and laboratory isoenzyme evidence of myocardial necrosis), cardiac failure, atrial fibrillation, and stroke. All complications occurred within 30 days or during a longer period if the patient was still in the hospital and were defined according to the joint Society of Thoracic Surgeons and European Society of Thoracic Surgeons standard definition of terminology and variables. ${ }^{15}$

The normal distribution of numeric variables was first assessed by the Shapiro-Wilk normality test. Numeric variables with normal distribution were tested by the unpaired Student $t$ test, and those without normal distribution were tested by the Mann-Whitney test. Categoric variables were tested by the chi-square test or, if the observations in at least 1 cell were less than 10, the Fisher exact test.

No variable had more than $5 \%$ incomplete data. Missing variables were imputed by using multiple imputation techniques.

To adjust for confounders, we developed risk-adjusting models to estimate cardiopulmonary morbidity and 30-day mortality. Several variables were tested for a possible association with these 2 outcomes by stepwise logistic regression analyses (age, sex, body mass index, forced expiratory volume in 1 second, carbon monoxide lung diffusion capacity, presence of underlying coronary artery disease, cerebrovascular disease, diabetes, performance score, duration of surgery). The regression analyses generated regression equations that were used to estimate the expected outcomes for each period (pre-ERP and ERP).

The following logit equation was used to calculate predicted cardiopulmonary morbidity:

$$
\begin{aligned}
-1.16+0.032 \text { Xage } & -0.013 X F E V 1-0.03 X D L C O \\
+ & 0.0054 \text { Xoperation time. }
\end{aligned}
$$

The following logit equation was used to calculate predicted 30-day mortality:

$$
\begin{aligned}
-2.566-0.0446 X D L C O & +0.563 X C A D+0.0116 \text { Xoperation time } \\
& -1.59 \text { Xfemale sex }
\end{aligned}
$$

The risk-adjusted postoperative cardiopulmonary morbidity or mortality rates were then calculated in each group by using the following equation: (observed postoperative outcome/predicted postoperative outcome) $\times$ average observed postoperative outcome of the overall population. The risk-adjusted postoperative outcome is the outcome, which is expected to be associated with that period (ERP or pre-ERP) if the case mix in that group was similar to the average case mix characteristics of the overall population in the study. The statistical tests were performed on the statistical software Stata 12.0 (StataCorp, LP, College Station, Tex).

\section{RESULTS}

Table 2 shows the baseline and surgical characteristics of the patients undergoing operation before and after the start of the ERP. The 2 groups had similar characteristics with the exception of a higher incidence of coronary artery disease $(22 \%$ vs $15 \%, P=.03)$ and a lower proportion of patients with a performance status greater than 1 in the ERP group ( $4.7 \%$ vs $9.1 \%, P=.05)$ compared with the pre-ERP one.

Table 3 summarizes the results of the comparison of outcomes between patients operated before and after ERP. The 2 groups had a similar postoperative length of stay (ERP median 5 days; interquartile range, 3-7 vs pre-ERP 4;

\begin{tabular}{|c|c|c|c|}
\hline Variables & $\begin{array}{c}\text { Before ERP } \\
\text { (365 patients) }\end{array}$ & $\begin{array}{c}\text { After ERP } \\
\text { (235 patients) }\end{array}$ & $\begin{array}{c}P \\
\text { value }\end{array}$ \\
\hline Age, y & $68.8(63-75)$ & $69.7(63-76)$ & .38 \\
\hline Sex male $(\mathrm{n}, \%)$ & $146(40)$ & $99(42)$ & .60 \\
\hline $\operatorname{BMI}\left(\mathrm{kg} / \mathrm{m}^{2}\right)$ & $27(24-30)$ & $27.3(24-30)$ & .96 \\
\hline FEV $1 \%$ & $89.2(73-106)$ & $89.2(74-105)$ & .60 \\
\hline DLCO $\%$ & $72.0(61-83)$ & $71(59-85)$ & .54 \\
\hline $\mathrm{CAD}(\mathrm{n}, \%)$ & $55(15)$ & $52(22)$ & .03 \\
\hline $\operatorname{CVD}(\mathrm{n}, \%)$ & $26(7.1)$ & $10(4.3)$ & .15 \\
\hline Diabetes (n, \%) & $41(11.2)$ & $26(11.1)$ & .95 \\
\hline ECOG PS >1 (n, \%) & $33(9.1)$ & $11(4.7)$ & .05 \\
\hline $\begin{array}{l}\text { Resection within upper } \\
\text { lobe }(\mathrm{n}, \%)\end{array}$ & $239(65.5)$ & $162(68.9)$ & .38 \\
\hline Right side of resection (n, \%) & $211(57.8)$ & $137(58.3)$ & .90 \\
\hline $\begin{array}{l}\text { Conversion to open surgery } \\
(\mathrm{n}, \%)\end{array}$ & $40(11)$ & $27(11.4)$ & .84 \\
\hline Operation time (min) & $150(120-180)$ & $150(120-195)$ & .27 \\
\hline
\end{tabular}

TABLE 2. Comparison of baseline and surgical characteristics of patients undergoing operation on before and after the start of enhanced recovery pathway

Numeric variables are expressed as medians and interquartile range. Categoric variables are expressed as numbers and percentages of total. ERP, Enhanced recovery pathway; $B M I$, body mass index; $F E V I$, forced expiratory volume in 1 second; $D L C O$, carbon monoxide lung diffusion capacity; $C A D$, coronary artery disease; $C V D$, cerebrovascular disease; ECOG PS, Eastern Cooperative Oncology group Performance Score.

interquartile range, 3-7, $P=.44$ ). A similar number of patients in the 2 groups failed to meet the target of 5 days postoperative hospital stay only (ERP $41 \%$ vs pre-ERP $35 \%$, $P=.15)$.

No differences were found in the incidence of cardiovascular and pulmonary complications (ERP $22.6 \%$ vs pre-ERP $22.4 \%, P=.98$ ) or pure pulmonary complications (ERP $17.9 \%$ vs pre-ERP $16.7 \%, P=.71$ ) between the groups.

In-hospital or 30-day mortality occurred in 9 patients of the ERP $(3.8 \%)$ and in 8 patients of the pre-ERP group $(2.2 \%)(P=.31)$. Ninety-day mortality rates also were similar between the 2 groups (ERP $4.7 \%$ vs pre-ERP $3.0 \%, P=.37$ ).

Finally, no differences were noted in terms of 30-day (ERP $7.2 \%$ vs pre-ERP $7.4 \%, P=.94$ ) or 90 -day readmission rates (ERP $9.8 \%$ vs pre-ERP $12.3 \%, P=.34$ ).

Because postoperative morbidity and mortality may be influenced by patient- and surgical-related characteristics, we constructed regression models to adjust the outcomes for these confounders. These models were then used to calculate risk adjusted morbidity and mortality in each group (see "Patients and Methods").

The risk-adjusted cardiopulmonary morbidity rates were similar in the 2 periods (ERP $21.9 \%$ vs pre-ERP $22.9 \%, z$ test $P=.76$ ), whereas the risk-adjusted 30 -day mortality was higher in the ERP period compared with the pre-ERP one $(3.6 \%$ vs $2.3 \%$, z test $P=.0004)$. 
TABLE 3. Comparison of outcomes between patients operated on before and after the start of enhanced recovery pathway

\begin{tabular}{lccc}
\hline \multicolumn{1}{c}{ Outcomes } & $\begin{array}{c}\text { Before ERP } \\
\text { (365 patients) }\end{array}$ & $\begin{array}{c}\text { After ERP } \\
(\mathbf{2 3 5} \text { patients) }\end{array}$ & $\begin{array}{c}\boldsymbol{P} \\
\text { value }\end{array}$ \\
\hline $\begin{array}{l}\text { Cardiovascular and } \\
\text { pulmonary complications } \\
\text { (n, \%) }\end{array}$ & $82(22.4)$ & $53(22.6)$ & .98 \\
$\begin{array}{l}\text { Pulmonary complications } \\
\text { (n, \%) }\end{array}$ & $61(16.7)$ & $42(17.9)$ & .71 \\
RF (n, \%) & $17(4.7)$ & $12(5.1)$ & .68 \\
Pneumonia (n, \%) & $52(14.2)$ & $32(13.6)$ & .95 \\
\hline Atelectasis (n, \%) & $23(6.3)$ & $17(7.2)$ & .52 \\
\hline AF (n, \%) & $33(9.0)$ & $19(8.1)$ & .84 \\
\hline In-hospital mortality (n, \%) & $8(2.2)$ & $6(2.6)$ & .78 \\
\hline 30-d mortality (n, \%) & $8(2.2)$ & $9(3.8)$ & .31 \\
\hline 90-d mortality (n, \%) & $11(3.0)$ & $11(4.7)$ & .37 \\
\hline 30-d readmission (n, \%) & $27(7.4)$ & $17(7.2)$ & .94 \\
\hline 90-d readmission (n, \%) & $45(12.3)$ & $23(9.8)$ & .34 \\
\hline Relative 90-d readmission & $18(4.9)$ & $6(2.6)$ & .20 \\
\hline (n, \%) & & & \\
\hline LOS (d) & $4(3-7)$ & $5(3-7)$ & .44 \\
\hline LOS >5 d (n, \%) & $128(35)$ & $96(41)$ & .15 \\
\hline
\end{tabular}

Numeric variables are expressed as medians and interquartile range. Categoric variables are expressed as numbers and percentages of total. ERP, Enhanced recovery pathway; $R F$, respiratory failure (need for mechanical ventilation for $>24$ hours or reintubation at any time postoperatively); $A F$, atrial fibrillation; $L O S$, length of stay.

\section{DISCUSSION \\ Main Findings}

In our specialty, there is limited evidence regarding the effectiveness of the ERP on outcome. In particular, there is no study specifically focusing on the application of the ERP in the context of minimally invasive thoracic surgery, which is by definition one of the main elements of the ERP. Therefore, we set up this retrospective analysis to compare the outcomes after VATS lobectomy before and after the start of the ERP in our institution. We were not able to find differences in incidence of cardiopulmonary complications, 30-day or 90-day mortality rates, length of postoperative stay, and hospital readmission within 30 or 90 days from the operation between the groups.

Extrapolation of our findings to the wider thoracic surgery community is limited because our data are a snapshot of a clinical practice and represent outcomes from a single thoracic center. However, our unit undertakes approximately 300 lung resection operations per year and has a high rate $(75 \%)$ of resection via the VATS approach. VATS is a recent development in lung resection surgery and may now be regarded as the recommended approach for early-stage lung cancer. ${ }^{16}$

Our findings suggest that all components of an ERP may not be necessary for patients undergoing VATS resection. An ERP inevitably increases cost; the introduction of patient diaries, incentive spirometry, carbohydrate loading, preoperative warming, nutritional supplements, and use of a dedicated ERP nurse cost $\$ 189.0$ per patient in our unit in 2016. In the absence of demonstrable benefit for patients, this extra cost is hard to justify in a modern healthcare system under financial strain.

There are 2 potential explanations of our findings. First, it is possible that our ERP interventions were insufficient to produce a measurable benefit. As can be seen in Table 1, there were several differences in perioperative care when comparing the pre-ERP and ERP patient groups, but also some elements common to both groups. Some of the common elements may be missing components from a traditional ERP, for example, use of epidural analgesia to avoid systemic opiates or use of goal-directed therapy to avoid fluid overload. However, we believe a more likely explanation is that the pre-ERP standard care already involved sufficient ERP components $s^{5}$ to provide patients undergoing VATS with good outcomes, for example, the preoperative information and health advice, antibiotic prophylaxis, minimally invasive surgery, single chest drain, digital drainage system, and multimodal analgesia, although variable. Identifying which ERP components are the most useful is challenging, and the literature does not provide answers. A large metaanalysis of ERP in various specialties was not able to identify any significantly beneficial individual components, ${ }^{2}$ and indeed the key to the success of any ERP may well be the synergistic effect of all elements being applied together by a unit used to managing patients in this way.

Second, VATS lung resection may be sufficiently noninvasive, low risk, and typically associated with uncomplicated recovery that the ERP is simply not required. The clinical experience of surgeons and anesthetists suggests that patients who have more major and high-risk surgical procedures, with the associated physiologic disturbances and stress responses to surgery, have poorer outcomes and so may need additional interventions in the perioperative period to help them overcome these challenges. The literature on the ERP does not support this view: Different surgical specialties have not been found to derive different degrees of benefit from the ERP. ${ }^{2}$ One study of patients undergoing VATS resection found a significant difference in the length of stay for patients before and after introduction of an ERP, ${ }^{17}$ but in this study the length of stay was very long ( 29 and 19 days in the 2 groups, respectively), suggesting that the patients differed significantly from our cohort ( 5 and 5 days, respectively). Thus, there is no current evidence to contest our findings that patients undergoing VATS do not benefit from or require the ERP.

\section{Study Limitations}

First, our analysis focused on a population of patients undergoing minimally invasive thoracic surgery. Generalizability of the results to patients undergoing open surgery 
needs to be verified by further work. As discussed earlier, VATS is a key element of the ERP and likely the one with the greatest influence on outcome. Comparing 2 groups of patients, both receiving this element, may blur the effect of the other ERP components on outcome. Also, the proportion of VATS compared with open cases may play a role and have an effect on the cost-effectiveness of the ERP. Our findings may not be generalizable to units with a lower proportion of minimally invasive cases compared with open cases. The relationship between VATS relative volume and ERP effectiveness warrants further investigations.

Moreover, the results generated by this study may not be applicable in units in which most of the ERP elements are not included in their standard care, as occurred in our setting. The implementation of ERP may be more beneficial and cost-effective in these institutions even for patients undergoing VATS.

Second, we reported only objective outcomes (morbidity and mortality). Patient-reported outcomes such as quality of life or patient satisfaction with care were not routinely collected in this group of patients. These are certainly additional end points requiring investigation in the future to evaluate the true success of ERP in this patient group. Although the inclusion of multiple ERP elements in the perioperative care of patients undergoing VATS did not provide any clinical advantage in terms of morbidity or mortality in our unit, we cannot rule out that those elements may have improved the overall patient experience with care compared with the pre-ERP period.

Another possible limitation is the relatively shorter ERP period compared with the pre-ERP period. Especially if adherence to the program was slow at the beginning, this may introduce a bias against ERP because of overlapping of pathways of care. To minimize this problem, we excluded those patients managed during the first month of ERP implementation (washout period). Because most of the ERP elements were already part of our standard care, this period was sufficient to fully implement ERP in our setting with optimal adherence to the program as shown in Table 1.

Finally, this is not a randomized study, and inherent problems of cohort selection may be present as in all retrospective investigations. We chose not to use balancing score and case matching in this context because the only variable that would affect the propensity of the patients to receive either intervention (ie, ERP or non-ERP) is whether they underwent their surgery before or after January 2016. The 2 groups were substantially similar in terms of baseline and surgical characteristics because patient selection criteria for surgery did not change during the relatively short study period. In any case, we used logistic regression analysis to adjust for the effect of other confounders and verify the independent influence of ERP on the outcomes.

\section{CONCLUSIONS}

For patients undergoing VATS lung resection, we may now be in a "post-ERP" world. It is possible that sufficient beneficial components of ERP are now received by all patients and that this should now be regarded as "standard" rather than "enhanced" care. Will it ever be possible to demonstrate outcome benefits to justify the extra cost of ERP for these patients? Progress in medical care, as in sports, is now mostly achieved by "aggregation of marginal gains," in which numerous small modifications result in improved outcome. If this proves to be the case for perioperative care bundles such as ERP, then the research needed to tease apart the marginal gains from so many small interventions in such diverse surgical procedures will be extremely challenging, and perhaps impossible. Future studies will be needed to confirm our findings in other centers with a different case mix of patients, different distribution of minimally invasive surgery, and different standard care pathways.

\section{Conflict of Interest Statement}

Authors have nothing to disclose with regard to commercial support.

The authors thank Angela Mandelj, ERP specialist nurse for our unit, for consistent and excellent clinical support of the patients and help with inputting the data used for this article.

\section{References}

1. Kehlet H, Wilmore DW. Evidence-based surgical care and the evolution of fasttrack. Ann Surg. 2008:248:189-98.

2. Nicholson A, Lowe MC, Parker J, Lewis SR, Alderson P, Smith AF. Systematic review and meta-analysis of enhanced recovery programmes in surgical patients Br J Surg. 2014;101:172-88.

3. Ljungqvist $\mathrm{O}$, Scott M, Fearon KC. Enhanced recovery after surgery: a review. JAMA Surg. 2017;152:292-8.

4. Fiore JF Jr, Bejjani J, Conrad K, Niculiseanu P, Landry T, Lee L, et al. Systematic review of the influence of enhanced recovery pathways in elective lung resection. J Thorac Cardiovasc Surg. 2016;151:708-15.

5. Brown LM. "Moving right along" after lung resection, but the data suggest "not so fast" J Thorac Cardiovasc Surg. 2016;151:715-6.

6. Zehr KJ, Dawson PB, Yang SC, Heitmiller RF. Standardized clinical care pathways for major thoracic cases reduce hospital costs. Ann Thorac Surg. 1998;66:914-9.

7. Wright CD, Wain JC, Grillo HC, Moncure AC, Macaluso SM, Mathisen DJ. Pulmonary lobectomy patient care pathway: a model to control cost and maintain quality. Ann Thorac Surg. 1997;64:299-302.

8. Salati M, Brunelli A, Xiume F, Refai M, Pompili C, Sabbatini A. Does fasttracking increase the readmission rate after pulmonary resection? A casematched study. Eur J Cardiothorac Surg. 2012;41:1083-7.

9. Muehling BM, Halter GL, Schelzig H, Meierhenrich R, Steffen P, SunderPlassmann L, et al. Reduction of postoperative pulmonary complications after lung surgery using a fast track clinical pathway. Eur J Cardiothorac Surg. 2008;34:174-80.

10. Zhuang CL, Huang DD, Chen FF, Zhou CJ, Zheng BS, Chen BC, et al. Laparoscopic versus open colorectal surgery within enhanced recovery after surgery programs: a systematic review and meta-analysis of randomized controlled trials Surg Endosc. 2015;29:2091-100.

11. Falcoz PE, Puyraveau M, Thomas PA, Decaluwe H, Hürtgen M, Petersen RH, et al. Video-assisted thoracoscopic surgery versus open lobectomy for primary non-small-cell lung cancer: a propensity-matched analysis of outcome from the European Society of Thoracic Surgeon database. Eur J Cardiothorac Surg. 2016;49:602-9. 
12. Burt BM, Kosinski AS, Shrager JB, Onaitis MW, Weigel T. Thoracoscopic lobectomy is associated with acceptable morbidity and mortality in patients with predicted postoperative forced expiratory volume in 1 second or diffusing capacity for carbon monoxide less than $40 \%$ of normal. J Thorac Cardiovasc Surg. 2014; 148:19-28.

13. Paul S, Altorki NK, Sheng S, Lee PC, Harpole DH, Onaitis MW, et al. Thoracoscopic lobectomy is associated with lower morbidity than open lobectomy: a propensity-matched analysis from the STS database. J Thorac Cardiovasc Surg. 2010;139:366-78.

14. Brunelli A, Kim AW, Berger KI, Addrizzo-Harris DJ. Physiologic evaluation of the patient with lung cancer being considered for resectional surgery. Chest. 2013;143:166S-90S.

15. Fernandez FG, Falcoz PE, Kozower BD, Salati M, Wright CD, Brunelli A. The Society of Thoracic Surgeons and the European Society of Thoracic Surgeons general thoracic surgery databases: joint standardization of variable definitions and terminology. Ann Thorac Surg. 2015;99:368-76.

16. Howington JA, Blum MG, Chang AC, Balekian AA, Murthy SC. Treatment of stage I and II non-small cell lung cancer: diagnosis and management of lung cancer, 3rd ed: American College of Chest Physicians evidence-based clinical practice guidelines. Chest. 2013;143(5 Suppl):e278S-313S.

17. Maruyama R, Miyake T, Kojo M, Aoki Y, Suemitsu R, Okamoto T, et al. Establishment of a clinical pathway as an effective tool to reduce hospitalization and charges after video-assisted thoracoscopic pulmonary resection. Jpn J Thorac Cardiovasc Surg. 2006;54:387-90.

Key Words: video-assisted thoracoscopic surgery, enhanced recovery, morbidity, mortality, lung resection 